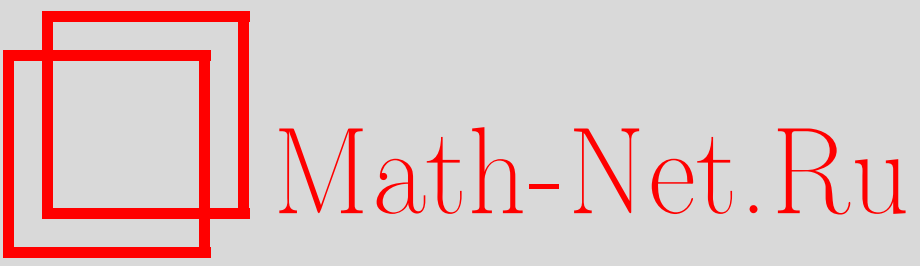

Г. В. Потёмин, О дифференциально-геометрических скобках Пуассона третьего порядка, УМН, 1997, том 52, выпуск 3, 173-174

DOI: https://doi.org/10.4213/rm857

Использование Общероссийского математического портала Math-Net.Ru подразумевает, что вы прочитали и согласны с пользовательским соглашением

http://www.mathnet.ru/rus/agreement

Параметры загрузки:

IP: 3.89 .185 .249

26 апреля 2023 г., 13:05:23 


\title{
О ДИФФЕРЕНЦИАЛЬНО-ГЕОМЕТРИЧЕСКИХ СКОБКАХ ПУАССОНА ТРЕТЬЕГО ПОРЯДКА
}

\author{
Г. В. Потёмин
}

В работе [1] определены однородные дифференциально-геометрические скобки Пуассона порядка $p, p \in \mathbb{N}$, и поставлена задача о классификации таких скобок. Для одной пространственной переменной скобки порядка $p$ имеют вид:

$$
\begin{gathered}
\left\{u^{i}(x), u^{j}(y)\right\}=g^{i j}(u(x)) \delta^{(p)}(x-y)+b_{k}^{i j}(u(x)) u_{1}^{k} \delta^{(p-1)}(x-y) \\
+\left[c_{k}^{i j}(u(x)) u_{2}^{k}+c_{k l}^{i j}(u(x)) u_{1}^{k} u_{1}^{l}\right] \delta^{(p-2)}(x-y)+\cdots \\
\cdots+\left[d_{k}^{i j}(u(x)) u_{p}^{k}+\cdots\right] \delta(x-y), \quad i, j, k, \ldots=1, \ldots, n, \\
u_{s}^{k}=D^{s} u^{k}, \text { где } D \equiv \frac{d}{d x} .
\end{gathered}
$$

Коэффиициентыпри $\delta^{(p-s)}$ имеют степень $s$, причем по определению $\operatorname{deg} f(u(x))=\operatorname{deg} u(x)=0$, $\operatorname{deg} \delta^{(q)}=\operatorname{deg} u_{q}=q, \operatorname{deg}(f g)=\operatorname{deg} f+\operatorname{deg} g$.

При $p=1$ получаем скобки Пуассона гидродинамического типа. По ним имеется обширная литература (см., например, [2]).

Дифференциально-геометрические скобки Пуассона определяют на многообразии $M^{n}$ полевых переменных $u^{i}$ своеобразную геометрию.

Класс дифференциально-геометрических скобок Пуассона порядка $p$ инвариантен относительно локальных преобразований переменных вида $u=u(v)$. При этом $g^{i j}$ преобразуются как компоненты тензора типа $(2,0)$, а величины $-C_{p}^{1} g_{j s} b_{k}^{s i},-C_{p}^{2} g_{j s} c_{k}^{s i}, \ldots,-C_{p}^{p} g_{j s} d_{k}^{s i}$, где $C_{p}^{m}-$ биномиальные коэффициенты, преобразуются как компоненты дифференциально-геометрических связностей [3].

В работе [4] С.П. Новиков высказал гипотезу, что “последняя" связность $\Gamma_{j k}^{i}$ в (1) симметрична и имеет нулевую кривизну. Эта гипотеза была доказана в [3], [5]. Тогда существует система координат, в которой связность $\Gamma_{j k}^{i}$ равна нулю. Эти локальные координаты определены с точностью до афффинных преобразований.

Невырожденные $\left(\operatorname{det}\left(g^{i j}\right) \neq 0\right)$ однородные дифференциально-геометрические скобки Пуассона второго порядка изучались в [3], [5], [6].

В специальной системекоординат скобки второго порядка приводятся к виду $\left\{u^{i}(x), u^{j}(y)\right\}=$ $\left(g^{i j} \delta^{\prime}(x-y)\right)^{\prime}$, где $g_{i j}=a_{i j k} u^{k}+g_{i j}^{0}, a_{i j k}$ и $g_{i j}^{0}$ - const и косимметричны по всем индексам [3], [5]. Скобка приводится к постоянному виду $\left\{u^{i}, u^{j}\right\}=g^{i j} \delta^{\prime \prime}(x-y)$, если и только если 2-форма $g_{i j} d u^{i} \wedge d u^{j}$ замкнута [6].

В работе [5] есть пример однородной дифференциально-геометрической скобки Пуассона третьего порядка, не приводящейся к постоянному виду. Пусть $\varepsilon=\left(\varepsilon^{i j}\right)$ - постоянная симметрическая матрица и $f=\left(c_{k}^{i j} u^{k}+d^{i j}\right)$ - невырожденны. Если выполнены условия $c_{i}^{k l} \varepsilon_{l_{j}}+$ $c_{j}^{k l} \varepsilon_{l i}=0, \varepsilon^{i e} c_{e}^{m j} \varepsilon_{m p} f^{p k}+$ (цикл $\left.i, j, k\right)=0$, то можно построить скобку Пуассона третьего порядка

$$
\left\{u^{i}(x), u^{j}(y)\right\}=\left(g^{i j} \delta^{\prime \prime}(x-y)+c_{k}^{i j} u_{1}^{k} \delta^{\prime}(x-y)\right)^{\prime},
$$

где $g^{i j}=\left(\varepsilon f^{-1} \varepsilon f^{-T} \varepsilon\right)^{i j}, c_{k}^{i j}=\left(\varepsilon f^{-1} \varepsilon \frac{\partial f^{-T}}{\partial u^{k}} \varepsilon\right)^{i j}$, причем $g_{, k}^{i j} \equiv \frac{\partial g^{i j}}{\partial u^{k}}=c_{k}^{i j}+c_{k}^{j i}$.

Рассмотрим дифференциально-геометрические скобки Пуассона третьего порядка в специально системе координат.

Теорема 1. Дифференциально-геометрические скобки Пуассона третьего порядка приводятся к виду (2), причем коэффичиенты в (2) должнны удовлетворять условия⿻․:

$$
\begin{gathered}
g_{, k}^{i j}=c_{k}^{i j}+c_{k}^{j i}, \quad c_{l}^{i j} g^{l k}=-c_{l}^{k j} g^{l i}, \\
c_{l}^{i j} g^{l k}+c_{l}^{j k} g^{l i}+c_{l}^{k i} g^{l j}=0, \quad c_{l, m}^{i j} g^{l k}=c_{l}^{i k} c_{m}^{l j}-c_{l}^{k i} c_{m}^{l j}-c_{l}^{k j} g_{, m}^{l i} .
\end{gathered}
$$


Условия (3) можно переписать для величин с нижними индексами: $c_{m n k}=g_{m j} g_{n i} c_{k}^{i j}$. Тогда (3) можно переписать следующим образом:

$$
\begin{aligned}
& g_{m n, k}=-c_{m n k}-c_{n m k}, \quad c_{m(n k)}=0, \\
& c_{[m n k]}=0, \quad c_{m n k, l}=-g^{p q} c_{p m l} c_{q n k} .
\end{aligned}
$$

Метрика $g_{m n}$ зависит квадратично от $u^{i}$ [3], [5]. Действительно, из (4) следует, что $c_{m n k}, l r=0$, T.e.

$$
g_{m n}=g_{m n p q} u^{p} u^{q}+g_{m n p} u^{p}+g_{m n}^{0} .
$$

Теорема 2. Метрика $g_{m n}$ и тензор кручения $T_{j k}^{i}=g^{i m} c_{m j k}$, связанные соотношениями (4), полностью определяют дифференциально-геометрическую скобку Пуассона третьего порядка.

Невырожденная дифференциально-геометрическая скобка Пуассона третьего порядка приводится локальной заменой координат $u=u(v)$ к постоянному виду $g^{i j} \delta^{\prime \prime \prime}(x-y)$, если и только если связность $\Gamma_{j k}^{i}=-\frac{1}{3} g_{j l} c_{k}^{l i}$ в (1) симметрична. Это всегда верно при $n=1$ [3], [5].

ПримеР. Дифференциально-геометрические скобки Пуассона третьего порядка встречаются при изучении уравнений ассоциативности в двумерной топологической теории поля [7]. Уравнения ассоциативности можно представить как систему гидродинамического типа: $u_{t}^{1}=u_{x}^{2}$, $u_{t}^{2}=u_{x}^{3}, u_{t}^{3}=\left(u^{2^{2}}-u^{1} u^{3}\right)_{x}$. Эта система бигамильтонова: $\left(u_{t}^{1}, u_{t}^{2}, u_{t}^{3}\right)^{T}=J_{0} \delta H_{0}=J_{1} \delta H_{1}$, где $\delta$ обозначает вариационную производную, $J_{0}$ - гамильтонов оператор первого порядка [7], а $J_{1}$ - оператор третьего порядка,

$$
J_{1}=\left(\begin{array}{ccc}
0 & 0 & D^{3} \\
0 & D^{3} & -D^{2} u^{1} D \\
D^{3} & -D u^{1} D^{2} & D^{2} u^{2} D+D u^{2} D^{2}+D u^{1} D u^{1} D
\end{array}\right) .
$$

Плотность гамильтониана $H_{1}=-\frac{1}{2} u^{1}\left(D^{-1} u^{2}\right)^{2}-\left(D^{-1} u^{2}\right)\left(D^{-1} u^{3}\right)$. Здесь метрика имеет вид: $d s^{2}=-2 u^{2}\left(d u^{1}\right)^{2}+2 u^{1} d u^{1} d u^{2}+2 d u^{1} d u^{3}+\left(d u^{2}\right)^{2}$. Если произвести замену $u^{1}=v^{1}$, $u^{2}=v^{2}-\left(v^{1}\right)^{2}, u^{3}=v^{3}+v^{1} v^{2}-\frac{1}{3}\left(u^{1}\right)^{3}$, то метрика станет плоской: $d s^{2}=2 d v^{1} d v^{3}+\left(d v^{2}\right)^{2}$. Но несмотря на это, оператор $J_{1}$ не сводится к оператору с постоянными коэффициентами, так как система координат $\left(u^{i}\right)$, где исчезает последняя связность, и система координат $\left(v^{i}\right)$, где метрика имеет постоянные коэффициенты, - различны. В [7] найдена замена переменных: $u^{1}=w_{x}^{1}$, $u^{2}=w_{x}^{2}, u^{3}=w_{x}^{3}-w_{x}^{1} w_{x}^{2}-w^{2} w_{x x}^{1}, w^{i}$ - плотности трех нелокалных функций Казимира гамильтонова оператора $J_{1}$. В новых переменных гамильтонов оператор $J_{1}$ преобразуется в $\tilde{J}_{1}=-\left(\begin{array}{lll}0 & 0 & 1 \\ 0 & 1 & 0 \\ 1 & 0 & 0\end{array}\right) D$.

\section{СПИСОК ЛИТЕРАТУРЫ}

[1] Дубровин Б. А., Новиков С. П. // Докл. АН СССР. 1984. Т. 279. № 2. С. 294-297. [2] Новиков С. П. // УМН. 1989. Т. 44. №6. С. 29-98. [3] Потёмин Г. В. Некоторые вопросы дифференциальной геометрии и алгебраической геометрии в теории солитонов // Дис. ... канд. физ.-матем. наук. М., 1991. [4] Новиков С. П. // УМН. 1985. Т. 40. № 4. С. 79-89. [5] Doyle P.W. // J. Math. Phys. 1993. V. 34. №4. P. 1314-1338. [6] Потёмин Г.В.// Докл. АН CCCP. 1986. Т. 286. №1. C. 39-42. [7] Ferapontov E. V., Galvao C. А.P., Mokhov O.I., Nutku Y. Bi-Hamiltonian structure of equations of associativety in 2-d topological field theory // Comm. Math. Phys. 1997. 